\title{
The Design of Wireless Snoring Monitoring System Based on NRF24L01
}

\author{
Jinjun Lu \\ School of Physics and Telecommunication Engineering, Shaanxi University of Technology, \\ Hanzhong, China \\ hsylu@163.com
}

\section{Keywords: Snoring sensors; nRF24L01; Wireless monitoring; MCU; Snoring monitoring}

\begin{abstract}
A portable wireless snoring monitoring system is designed. The system consists of a slave and a host, in which the slave plays the roles of data collecting, processing and sending, and the host undertakes the functions of data reception, analysis, storage and touch display. The design can effectively makes up some shortcomings of the current professional medical snore monitoring, and also fills the market blank of the home snore monitoring equipment. The test results indicate that the system has high accuracy, stability and practicability, and thereby can be widely promoted.
\end{abstract}

\section{Introduction}

Snoring medically known as SAS (sleep apnea syndrome), is a condition of sleep disorders that stop breathing while sleeping. A study can establish reliable indices of the disorder, derived from the number and type of event per hour of sleep (Apnea Hypopnea Index (AHI), or Respiratory Disturbance Index (RDI)), associated to a formal threshold, above which a patient is considered as suffering from sleep apnea, and the severity of their sleep apnea can then be quantified. Mild OSA (Obstructive Sleep Apneas) ranges from 5 to 14.9 events per hour, moderate OSA falls in the range of 15-29.9 events per hour, and severe OSA would be a patient having over 30 events per hour. SAS repeatedly pause sleep breathing, causing severe hypoxia in the brain, the formation of hypoxemia, and induced hypertension, arrhythmia, myocardial infarction, angina. More serious is the nighttime breathing pauses for more than 120 seconds prone to sudden death in the early morning.

For this reason, the snoring monitoring and qualitative judgments seem to be important, the traditional hospital for sleep and snoring with overnight "polysomnography (PSG)". PSG monitors many body functions including brain, eye movements, muscle activity or skeletal muscle activation and heart rhythm during sleep. It is usually performed at night, when most people sleep, though some labs can accommodate shift workers and people with circadian rhythm sleep disorders and do the test at other times of day. PSG monitoring takes at least 45-60 minutes, in the meantime, a large number of sensors need to be worn on the patient by this method, which seriously affects the patient's sleep. Moreover, if the sensor drops off during use, it needs to be measured again.

This paper introduces a method of portable wireless snore monitoring system. The slave of the system completes the data acquisition and data processing and sending functions, the host can receive, analyze, store and display the data. The system has good accuracy, stability, practicality, simple operation, suitable for snoring sound monitoring in the home environment.

\section{System Function Overview}

The system consists of a master and a slave. The slave to signal acquisition, amplification, filtering, and the processed data is then sent to the host through nRF24L01 wireless module. The host determines the condition of the user's sleep apnea according to the medical definition after receiving the information of time and temperature when the data is received and analyzed by the nRF24L01. The system displays the result and stores the data on an SD card, which can be called up in the future as needed. System block diagram shown in Fig. 1 


\section{System Hardware Design}

The system hardware design ideas mainly follow the points: (1) Low power consumption, so the monitoring device host and slave are using 3.3V power supply system. (2) High stability and reliability, so most of the hardware systems are used in current applications has been relatively mature chip, such as the slave used the STC12LE5206AD chip. (3) Small size, because the slave to be worn on the patient, and cannot affect the patient's sleep, so the system components are used chip components. (4) The system should be ergonomically designed to meet the operating habits and needs of most people. Therefore, the system strives to achieve the current mainstream operation mode, which can be reflected in the system man-machine exchange equipment using TFT2.4 color touch screen.

Signal Acquisition and Processing Circuit. Studies have shown that the maximum frequency of human

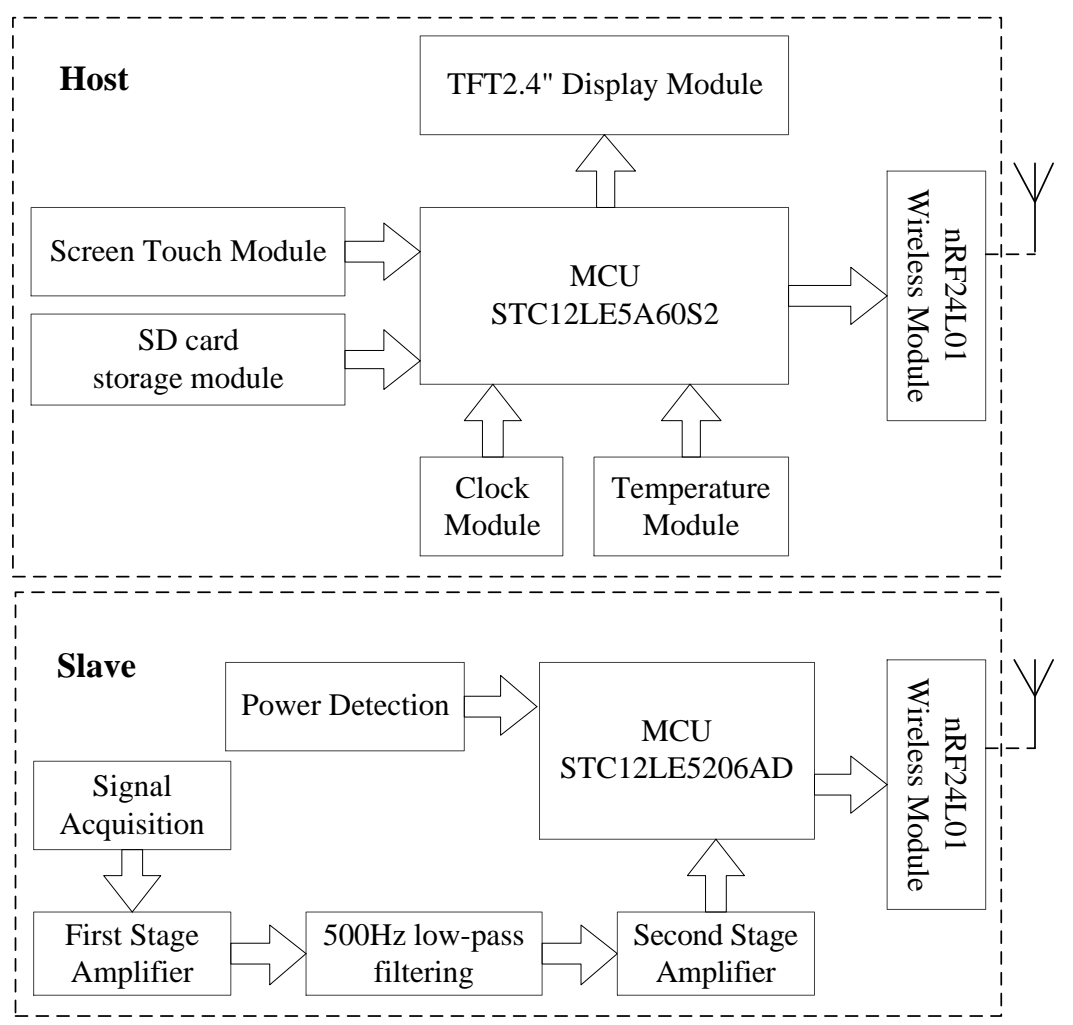

Figure 1. System block diagram

snoring does not exceed $400 \mathrm{HZ}, 500 \mathrm{HZ}$ low-pass filter after the experiment verified that the system requirements, both effectively remove the signal interference, but also ensure that do not lose useful signals, in order to make the low-pass filter effect is more ideal, so in the first low-pass filter signal amplification. After a stage of amplification and low-pass filtered signal after two amplification, useful signal at this time the maximum amplitude greater than $3.0 \mathrm{~V}$ less than $3.3 \mathrm{~V}$. Signal acquisition using a professional HS-3 type snoring sensor.

The first stage and the second stage amplifier circuit are the same direction proportional amplifier circuit with high input resistance and low output resistance. The op amp uses the LMV358, a general purpose low voltage rail-to-rail op amp that operates from a single supply at $3.3 \mathrm{~V}$ system voltage to meet system needs. It is a single power supply, so in a first amplifier circuit needs to add a bias circuit to provide virtual ground circuit to prevent reverse cut-off distortion.

Due to the characteristics of the sensor will introduce about $3 \mathrm{KHz}$ interference, at the same time as the human snoring frequency is not higher than $400 \mathrm{HZ}$. Therefore, in order to remove the interference signal and retain the effective signal at the same time, the system adopts the $500 \mathrm{~Hz}$ low pass filter, which makes the system achieve the purpose of high stability.

Host and Slave Wireless Communication Circuit. The wireless module used for the 
communication between the host and slave of the system adopts the nRF24L01, which has the characteristics of receiving and transmitting unity, low working voltage, low power consumption and strong anti-interference ability. This is a work in the $2.4 \sim 2.5 \mathrm{GHz}$ world's common ISM band microcontroller wireless transceiver chip. Wireless transceivers include: frequency generator, enhanced SchockBurst mode controller, power amplifier, modulator and demodulator. We can set its output power through the SPI interface, channel selection and protocol settings. Very low current consumption, which operates at transmit power of $-6 \mathrm{dBm}$ in transmit mode, consumes $9 \mathrm{~mA}$, $12.3 \mathrm{~mA}$ in receive mode, and consumes less power in power-down and standby modes. These features conform to the requirements of the low power consumption of the system. The wireless module is directly connected with the MCU I/O port, and the data interaction is completed by simulating the time sequence of the SPI bus.

Host Clock Module and Temperature Module Circuit. In order to achieve the system design of home-based, practical features, design joined the calendar and thermometer functions in the system circuit, while the calendar is the only reference for system operation. The thermometer uses the DS18B20 chip and the calendar uses the DS1302 chip. The thermometer uses DS18B20 chip and the calendar uses DS1302 chip, which DS18B20 circuit design is simple, only one using the pull-up resistor data line, and DS1302 in order to make it can continue to run in the system power-down need to clock chip plus power supply, the power supply with $3 \mathrm{~V}$ button battery.

System PCB Production. After the circuit was successfully debugged on a breadboard, double-layer PCBs were drawn using Altium Designer 10.0 as designed. From the space-saving point of view, all the components of the host and slave devices are used in the chip package.

\section{System Software Design}

Host and slave programs using $\mathrm{C}$ language design, easy to maintain. The programs are all encapsulated in packages, with good reliability. PC part, the program uses LabVIEW software to complete, the use of graphical language, with a concise program, feature-rich features.

Slave Part Software Design. When the slave starts working, first delay $2 S$, the system initialization of all modules, while waiting for $\mathrm{AD}$ conversion voltage reference stable, so that the subsequent data processing more accurate. Followed by signal acquisition, acquisition program is first of all the battery power system through the $\mathrm{AD}$ conversion, separate preservation. Then the signal collected by the HS-3 sensor is directly connected to the single chip microcomputer for AD conversion. The converted signal enters the signal processing stage. Because the converted data is chaotic, the converted signal needs to be filtered. Filtering is the first method used to compare the local maximum, in short, is to take a maximum of every thirty data as a representative of these data, the rest of the data discarded. Then use a smoothing filter to filter the data, the use of queue FIFO, build a capacity of 28 per queue into a data average, as the data output. Queue capacity selection based on the larger capacity of the smoother curve of the data curve, but the more sluggish the system response, otherwise the opposite rule. The reason why the smoothing filter is used to filter the data is because the smoothing filter can eliminate the instantaneous error while filtering and can strengthen the energy of the small and small signal, which is good for judging. Then the processed every 30 plus a system power, 31 data package to send.

Host Part Software Design . Host software complexity is far higher than the slave, in order to maintain convenience and clear thinking, so the host program uses a modular design, the system needs to be used only need to call again on the line. The host of the main program flow chart shown in Fig. 2 


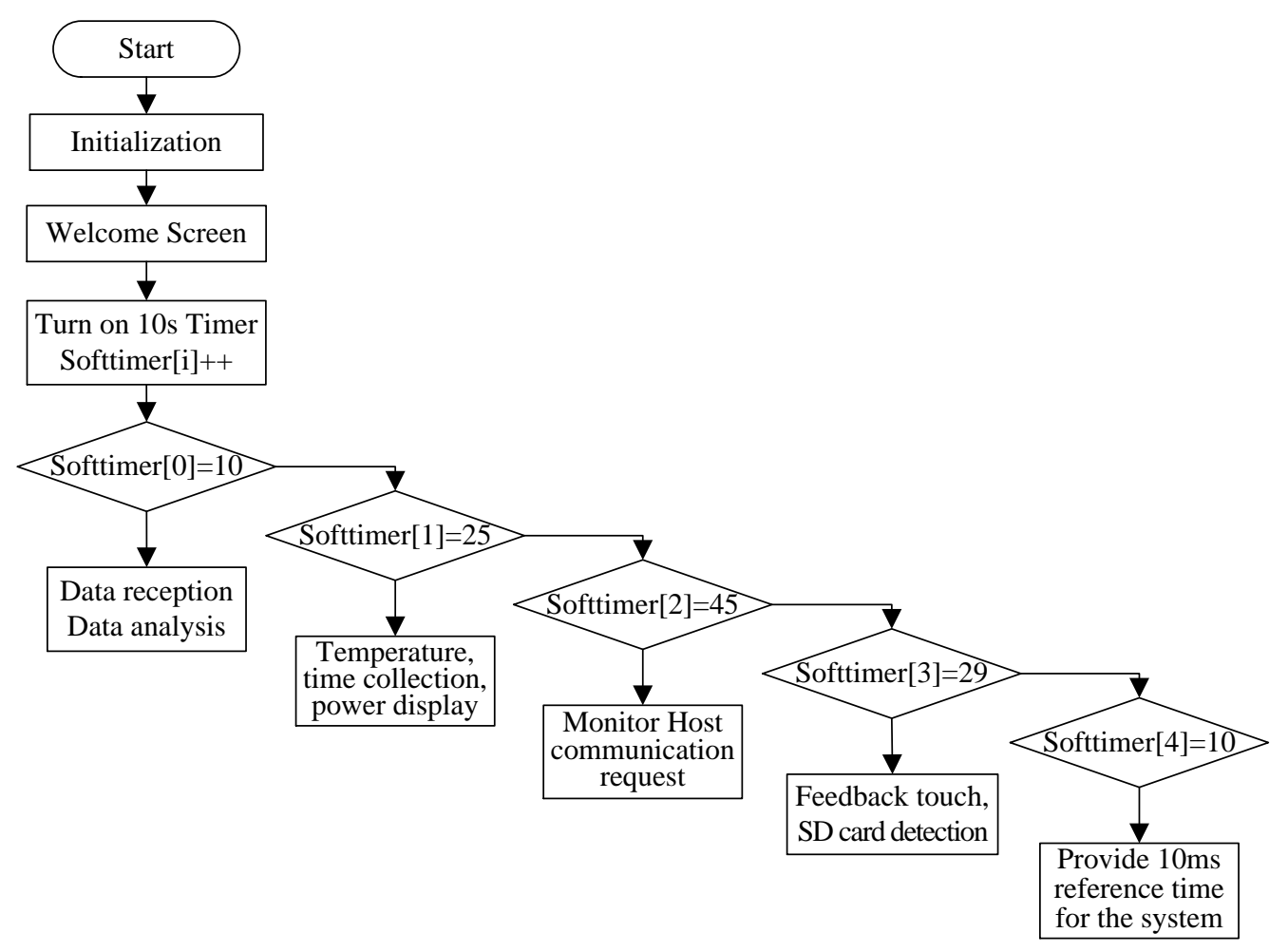

Figure 2. Host software flow chart

The flowchart shows that after the host is powered on, the system first initializes the hardware and software to be used in the program. System need to initialize the module, timer, serial port, SD card, NRF24L01 wireless module, TFT2.4-inch color display, touch screen SPI, clock, temperature module and so on. The system then shows a two-second welcome screen. More modules in the system, and the difference between each module priority is relatively large for the system, so the program will be on the module in the timer interrupt. According to the system requirements, after the system displays the welcome screen, the 5 -channel $10 \mathrm{~ms}$ timer is on, and the system really starts to work. The importance of each module to the system determined experimentally, once every $100 \mathrm{~ms}$ received from the slave back to the data, this time there will be no packet loss phenomenon, and the slave sends data and the host to receive the basic synchronization data. Once every $250 \mathrm{~ms}$ temperature acquisition, the system needs to display all the parameters displayed. Communication between the host and the slave is relatively small, so every $450 \mathrm{~ms}$ on the host to request a communication signal monitoring, if the request signal is found, the slave to send data to the host computer. Relative to other modules, SD card and touch module use frequency is not high, after the experiment to determine the two modules every $290 \mathrm{~ms}$ to monitor and respond to meet the system requirements. In the snoring and other data processing signal needs timing processing, the system uses $100 \mathrm{~ms}$ as the reference time.

System and PC Communication Software Design. The communication between system and computer is using serial communication which follows the RS232 protocol. Through the configuration of the serial port register SCON, make the serial port work in mode one, the communication data format is 10 bits per frame, including 1 start bit, 8 data bits and 1 stop bit, the baud rate is set as 9600 . The main program flowchart shows that the serial communication monitoring once every $450 \mathrm{~ms}$, when receiving the communication request, first received by the host to send the four-frame query date information. The data bit sent by the serial port is ASCII code, so it is first converted into integer data, and then the four constants are converted into the date information according to the sending order. Each inquiry date corresponds to a single sector on the SD card, so at this moment, the corresponding data is found on the SD card according to the inquiry date information. Each monitoring will produce 21 data and then send out the 21 data cycle to complete the upper and lower computer communication. The flow chart is shown in Fig. 3 
Clock Module and Temperature Module Software. In order to achieve the system design of home-based, practical features. In the design process by adding a thermometer and calendar features, when the device does not perform snoring monitoring can be used as ordinary household products. Perpetual calendar in the realization of the date display, but also for the system to provide a clock reference. The temperature is obtained through the digital temperature chip DS18B20, the program mainly solves the problem of temperature acquisition and display. Perpetual calendar program is mainly to solve the problem of obtaining clock data, display and time setting. Fig. 4 shows the software design flow of the clock module and temperature module.

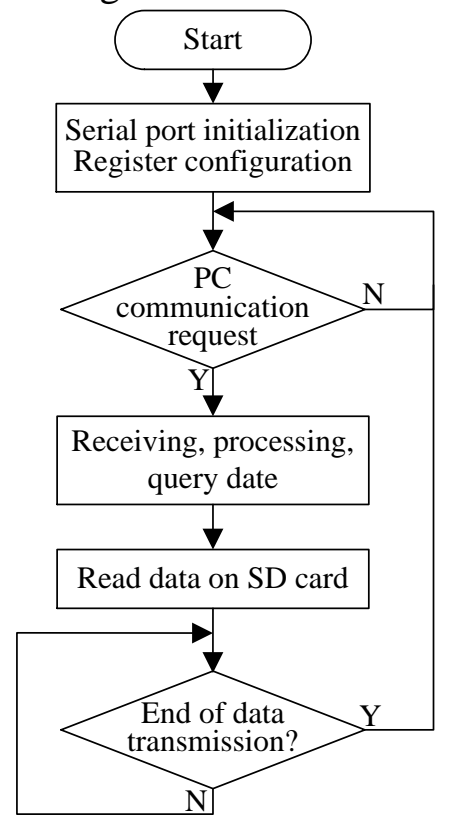

Figure 3. Host and slave communication flow chart

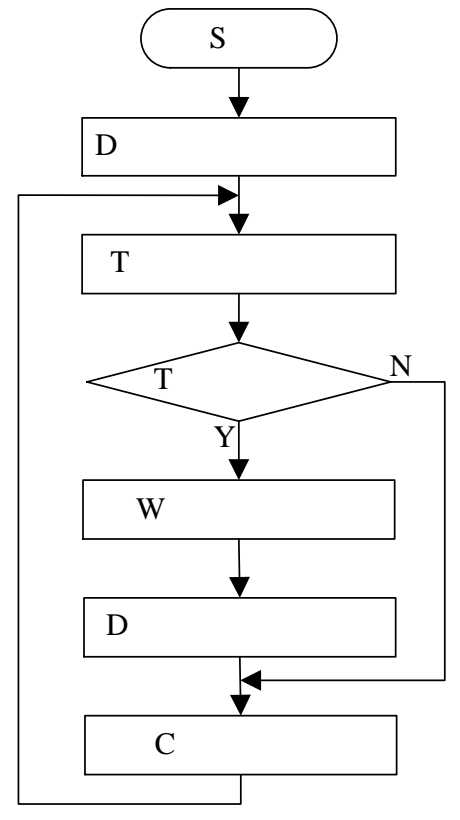

Figure4. Temperature module and clock module software flow chart

PC Software Design. PC software design using graphical language $G$ language, the development platform is LabVIEW software of company NI. The protocol to be followed when communicating between the PC and the host is the RS232 serial protocol, whereby the VISA resources in the serial VIs and functions can be used when designing the program. First, the VISA serial port configuration, the "Enable Termination $(\mathrm{T})$ " must be changed to "Disable (F)", otherwise it may result in incomplete data reception, the baud rate set at "Integer Input ", so that the baud rate can be changed, the other options by default. Then set the data buffer, because the former request to the latter fewer characters, so do not have to set the send buffer between the PC and host communication, just set the receive buffer. When the PC did not send a communication request or did not receive data sent by the host, the program has been executing the request to send and receive receipts until the arrival of valid data. Because the serial port to send data is sent in accordance with the ASCII code, so before the display on the data with the "string to byte array conversion" control conversion and combination. Then the data can be displayed or plot to complete all the features of the host computer.

\section{System Debugging and Testing}

Signal Processing Circuit Debugging. Signal processing circuit debugging is divided into three steps: (1) the theoretical verification under the Multisim software to test the theoretical calculation of the circuit design error and performance good or bad. (2) When the first step is successful, then build the circuit on the breadboard and use the oscilloscope and function signal generator to simulate the real signal for actual debugging. (3) Welding circuit connected to the actual signal, the actual debugging and circuit performance testing, according to the actual test results on the circuit to make minor adjustments. 
It is known from the above that the first stage and the second stage amplify the circuit principle is the same, so the amplifying circuit only carries on the debugging once. In accordance with the steps of this debugging, first in Multisim simulation, and then build a breadboard circuit, the signal generated by the function of the simulation signal generator, the oscilloscope to observe the input signal and the output signal, and software simulation magnification is slightly different . This small difference is caused by component errors, so no adjustment for the circuit. Press this circuit after welding, connected to the actual signal parameters are good, in line with system requirements.

$500 \mathrm{~Hz}$ low-pass filter is a second-order low-pass filter, set up the circuit simulation. AC signal from the small signal shows that the stop band, quality factor and other parameters are in line with the requirements. After the function signal generator and oscilloscope simulation, the results meet the requirements, set up the circuit, the circuit is working properly.

System Whole Hardware Debugging. The successful debugging of the circuit module alone, according to the order of signal transmission, the output signal is finally displayed through the oscilloscope. Enter the snoring signal from the HS-3 sensor and find that the maximum waveform on the oscilloscope does not exhibit cut-off distortion and is close to the supply voltage. This shows that the signal processing circuit meets the system requirements.

Overall System Debugging. After the overall system is successfully built, the whole system is debugged many times. Now choose one as an example, on January 17, 2018 in the laboratory environment test, the actual snoring 18 times, due to the system default to start using more interference, so after snoring three times to start recording, the actual record 15 times the success rate of $100 \%$. And the PC and connected to the host, display, calculate the parameters and the same host. System work is stable and good.

\section{Summary}

The portable wireless snoring monitoring system has a novel design, high sensitivity, friendly interface, easy operation, good portability, suitable for home use. This monitoring system fills the market gap in the monitoring of snoring products for civilian medical products and also makes up for some of the shortcomings of medical products. The system uses wireless communication, with a high non-awareness, the user can do long-term non-interference monitoring, so as to get the general snoring law. Analyze the severity of the user snoring and whether lesions occur. The data obtained by the system can also be used as a reference for the clinical diagnosis of the hospital. The system has a wide range of applications in the modern life of snoring so common that it can be widely promoted.

\section{Acknowledgements}

Supported by the National Natural Science Foundation of China under Grant Nos 11705113.

\section{References}

[1] Yeh-Liang Hsu;Ming-Chou Chen;Chih-Ming Cheng;Chang-Huei Wu.DEVELOPMENT OF A PORTABLE DEVICE FOR HOME MONITORING OF SNORING[J].Biomedical Engineering. Vol.17(2005):176-180.

[2] E.Z. OSMAN1;J. OSBORNE1;P.D. HILL2;B. W. V. LEE2.Snoring assessment: do home studies and hospital studies give different results?[J].Clinical Otolaryngology and Allied Sciences. Vol.23(1998): 524-527.

[3] C.Guo: The Wearable Sleep Monitoring System Based on Piezoelectric Film Sensors (M.Eng., Jilin University, China 2017), p.26.(In Chinese)

[4] Hida W;Kurosawa H;Miki H;Go T;Shindoh C;Kikuchi Y;Takishima T;Shirato K..Portable home monitoring system in screening for sleep-disordered breathing[J].Nihon Kyobu Shikkan Gakkai Zasshi.1995:46-49.

[5] Koja S;Arakaki H;Ogura C..Developing the portable type sleep apnea detector, and verifying 
the usefulness of the device[J].Seishin Shinkeigaku Zasshi. Vol.99(1997):181-197.

[6] Mlynczak M, Migacz E, Migacz M, et al. Detecting breathing and snoring episodes using a wireless tracheal sensor - a feasibility study[J]. IEEE Journal of Biomedical \& Health Informatics, 2016, PP(99):1-1.

[7] Y.Yuan: Research of Portable Sleep Monitoring and Analysis System (M.Med.,Nanjing Medical University,China 2010), p.12.(In Chinese)

[8] Furukawa T, Nakano H, Yoshihara K, et al. The Relationship between Snoring Sound Intensity and Morning Blood Pressure in Workers[J]. Journal of Clinical Sleep Medicine, Vol. 12(2016):1601-1606.

[9] Sankari A, Pranathiageswaran S, Maresh S, et al. Characteristics and Consequences of Non-apneic Respiratory Events during Sleep[J]. Sleep, Vol. 40(2017).

[10]Chen C M, Zhang X C, Li L I, et al. Effect of nursing intervention in monitoring of multi sleep breathing $[\mathrm{J}]$. Cardiovascular Disease Journal of Integrated Traditional Chinese \& Western Medicine, 2017. 